\title{
Direct generation of linearly polarized photon emission with designated orientations from site-controlled InGaN quantum dots
}

\author{
Anders Lundskog ${ }^{1}$, Chih-Wei Hsu ${ }^{1}$, K Fredrik Karlsson ${ }^{1}$, Supaluck Amloy ${ }^{1,2}$, Daniel Nilsson ${ }^{1}$, Urban Forsberg ${ }^{1}$, \\ Per Olof Holtz ${ }^{1}$ and Erik Janzén ${ }^{1}$
}

Semiconductor quantum dots (QDs) have been demonstrated viable for efficient light emission applications, in particular for the emission of single photons on demand. However, the preparation of QDs emitting photons with predefined and deterministic polarization vectors has proven arduous. Access to linearly polarized photons is essential for various applications. In this report, a novel concept to directly generate linearly-polarized photons is presented. This concept is based on InGaN QDs grown on top of elongated GaN hexagonal pyramids, by which the predefined elongation determines the polarization vectors of the emitted photons from the QDs. This growth scheme should allow fabrication of ultracompact arrays of photon emitters, with a controlled polarization direction for each individual emitter.

Light: Science \& Applications (2014) 3, e139; doi:10.1038/Isa.2014.20; published online 31 January 2014

Keywords: GaN; InGaN; photoluminescence; polarized emission; quantum dot

\section{INTRODUCTION}

Quantum dots (QDs) have validated their important role in current optoelectronic devices and they are also seen promising as light sources for quantum information applications. An improved efficiency of laser diodes and light-emitting diodes can be achieved by the incorporation of QDs ensembles in the optically active layers. ${ }^{1}$ In addition, the proposed quantum computer applications rely on photons with distinct energy and polarization vectors, which can be seen as the ultimate demand on photons emitted from individual QDs. ${ }^{2}$ A common requirement raised for several optoelectronic applications, e.g., liquid-crystal displays, three-dimensional visualization, (bio)-dermatology ${ }^{3}$ and the optical quantum computers, ${ }^{4}$ is the need of linearly polarized light for their operation. For existing applications, the generation of linearly polarized light is obtained by passing unpolarized light through a combination of polarization selective filters and waveguides, with an inevitable efficiency loss as the result. These losses can be drastically reduced by employment of sources, which directly generate photons with desired polarization directions.

Conventional QDs grown via the Stranski-Krastanov (SK) growth mode are typically randomly distributed over planar substrates and possess different degrees of anisotropies. The anisotropy in strain field and/or geometrical shape of each individual QD determines the polarization performance of the QD emission. Accordingly, a cumbersome post-selection of QDs with desired polarization properties among the randomly distributed QDs is required for device integration. ${ }^{5}$
Consequently, an approach to obtain QDs with controlled site and polarization direction is highly desired.

Although no perfect solution has been presented yet, several approaches on the site and polarization control of QDs have been reported. Preferential nucleation sites with finite areas created by in situ electron beam lithography ${ }^{6}$ and prefabricated pyramidal ${ }^{7-11}$ or nanowire ${ }^{12-14}$ templates have been demonstrated to obtain site-controlled QDs. However, these approaches do not provide any obvious path to polarization control. One route to achieve polarization control of the QD-based light-emitting sources is to grow stacked SK QDs. ${ }^{15,16}$ Large stacks of SK QDs are analogous to quantum wires, with the strongest confinement in the lateral directions, in contrast to a thin QD for which the vertical confinement is more significant. The observed polarization of the emission is successively rotated from a predominant transverse-electric mode to a transverse-magnetic mode, as the number of QDs in the stack increases. However, the emission is relatively broad and it red-shifts significantly when the polarization is rotated from transverse-electric to transverse-magnetic due to the reduced vertical quantum confinement. ${ }^{15}$ The QD-based single photon sources with polarization control presented up to date relies on a dot-in-microcavity design, in which the QD emission has to be tuned in order to efficiently couple with cavity modes. The tuning of the QD emission with respect to the cavity resonance can be done by temperature ${ }^{17,18}$ or by taking advantage of the Stark effect in an electrically gated cavity. ${ }^{19}$ The concept with single QDs embedded in a 
nanowire is a promising alternative, as both the QD site and the polarization direction can be controlled. ${ }^{14}$ However, the polarization will be restricted to the direction parallel to the long axis of nanowire, as the mechanism of polarization control in this case is due to the large dielectric constant contrast between the nanowire and the surrounding air, attenuating the electric field transverse to the long axis of the nanowire. $^{14}$

In this work, an approach to directly generate linearly polarized QD emission is demonstrated. Deterministic polarization directions are achieved by introducing site-controlled InGaN QDs on top of elongated GaN-based hexagonal pyramids (GaN EHPs) without employing complex cavity solutions. The polarization directions of the QD emission are found to be well aligned with the orientation of the EHPs. The reliability and consistency for this architecture are tested by a statistical analysis of InGaN QDs grown on GaN EHP arrays with different in-plane orientations of the elongations.

III-nitrides (III-N) QDs with potential to emit from the deep ultraviolet to the infrared have emerged as one of the most technologically important materials for optoelectronic applications. The wide bandgap range of III-N semiconductors opens the possibility to attain deep confinement potentials for the QDs, which has potential for high-temperature operation, as already demonstrated for a GaN-based singlephoton source operating at $200 \mathrm{~K} .{ }^{20}$ Moreover, III-N QDs are seen to be the best candidate for high performance optoelectronic applications due to their feasibility of achieving QD emission with an inherently high degree of linear polarization. ${ }^{21}$ Our results clearly show that it is possible to control the polarization direction of the emitted photons from InGaN QDs by varying the orientations of the underlying $\mathrm{GaN}$ EHPs. The polarization direction of the ground-state-related emission from the QDs reflects the axis of in-plane anisotropy of the confining potential, concerning both strain and/or QD shape. ${ }^{22,23}$ The same polarization direction for different QDs indicates that all grown QDs possess the same unidirectional in-plane anisotropy. Such simultaneous alignment of multiple QDs with controllable orientations has not been reported up to now to the best of our knowledge.

\section{MATERIALS AND METHODS}

Patterned substrate for the growth of GaN EHPs and symmetric hexagonal pyramids (SHPs)

The GaN EHPs and ordinary sixfold SHPs were selectively grown through oval and circular openings, respectively, on a lithographically patterned SiN-masked substrate. The substrates consisted of a $2.0 \mu \mathrm{m}$ thick (0001)-oriented GaN film epitaxially grown on a 4 -inch, n-type, on-axis $4 \mathrm{H}-\mathrm{SiC}$ substrate starting with an AlN nucleation layer $(\sim 100 \mathrm{~nm}) .^{24}$ The defined pattern was created by conventional ultraviolet lithography and reactive ion etching technique. Both circular openings and oval openings were repeated to form $10 \times 10$ arrays with a center-to-center distance of $6.3 \mu \mathrm{m}$. The radii $(R)$ of the circular openings were made $1.5 \mu \mathrm{m}$. The oval openings were deviations from circular openings (Figure 1a) in such a way that a rectangle with a constant width of $2 R$ and variable lengths $E(E=0.2,0.4,0.6,0.8$ and $1 \mu \mathrm{m})$ was inserted between two semicircles along designated directions. An angular parameter $\alpha$, defined as the angle between the $E$-axis and the $[2 \overline{1} \overline{1} 0]$ direction of the underlying GaN template, was introduced to describe the orientations of GaN EHPs as shown in Figure $1 \mathrm{~b}$. $\alpha$ was varied in $30^{\circ}$ steps over a $150^{\circ}$ period giving rise to six different orientations for the oval openings. For simplicity, the GaN EHPs described below have $E=1.0 \mu \mathrm{m}$ and $\alpha=0^{\circ}$ unless indicated otherwise.

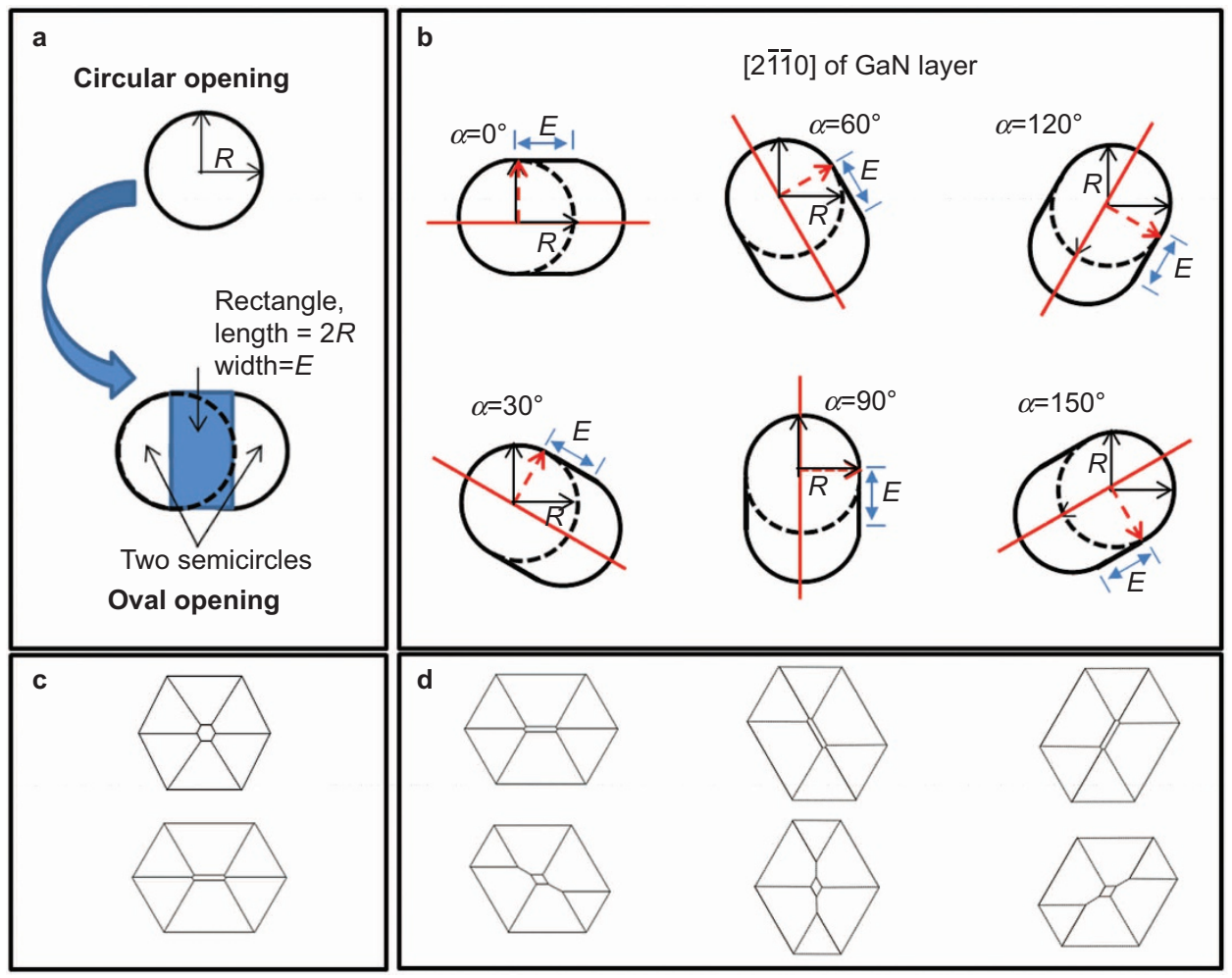

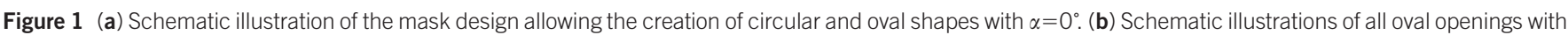

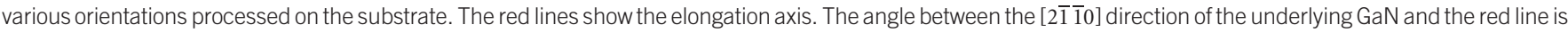

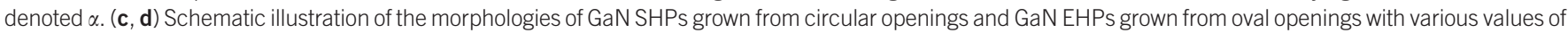
$\alpha$. EHP, elongated hexagonal pyramid; SHP, symmetric hexagonal pyramid. 
The growth conditions of GaN/InGaN/GaN EHPs (SHPs)

A horizontal low pressure hot-wall metal organic chemical vapor deposition system was used to grow entire structure. Trimethylgallium, trimethylindium and ammonia were used as precursors and the pressure during growth process was kept at 50 mbar. The growth conditions of the GaN EHPs and SHPs were based on our earlier development of uniform GaN SHPs with top (0001) facets. ${ }^{25}$ After the formation of GaN EHPs (SHPs), InGaN and GaN-cap layers were subsequently deposited on top of the EHPs (SHPs). The growth temperature for the GaN EHPs (SHPs) and the subsequent InGaN QWs/QDs were $1030{ }^{\circ} \mathrm{C}$ and $800{ }^{\circ} \mathrm{C}$, respectively. Upon this structure, a thin $\mathrm{GaN}$ cap layer (about 6-8 $\mathrm{nm}$ ) was grown at $800{ }^{\circ} \mathrm{C}$.

\section{Optical characterizations}

A continuous wave laser operating at $266 \mathrm{~nm}$ was used as the excitation source. The laser was focused to a spot diameter of $\sim 1.5 \mu \mathrm{m}$ through a reflective objective. The spot diameter was significantly smaller than the interdistance between the pyramids $(6.3 \mu \mathrm{m})$ and consequently could the emission properties of individual EHPs be studied. The emission was collected by the same objective and subsequently guided through the optics for polarization analysis, consisting of a rotatable half-wave retardation plate and a fixed linear polarizer, before entering the monochromator. This polarization analyzer provides detection of light linearly polarized along an adjustable angle $\theta$. In order to correlate the direction of the linearly polarized emission with the designated elongation direction, the reference angle $\theta=0^{\circ}$ corresponds to $\alpha=0^{\circ}$, which is parallel to the $[2 \overline{1} \overline{1} 0]$ direction of the underlying $\mathrm{GaN}$ template. The polarization direction of the emission $(\varphi)$ can be determined by fitting the measured data with the conventional formula for polarization: $I(\theta)=A+B \cos ^{2}(\theta-\varphi)$. To elucidate the polarization guiding effect $(\alpha=\varphi)$ in our work, an overall tolerance of $\pm 10^{\circ}$ was used in order to include all possible systematic errors, including the misalignment of the sample and the optics as well as the judgment of the corresponding $\theta$ for maximum and minimum intensities. Therefore, we consider the results satisfying $|\alpha-\varphi| \leq 10^{\circ}(\alpha \cong \varphi)$ to be consistent with polarization guiding. The cathodoluminescene (CL) was performed at $4 \mathrm{~K}$ in a Leo 1550 SEM equipped with a MonoCL2 system. The CL measurements were done by an electron beam with an accelerating voltage of $2.5 \mathrm{keV}$ and a spectral bandpass of $\sim 3 \mathrm{~nm}$ (for $2 \mathrm{~mm}$ slit and 1800 lines $\mathrm{mm}^{-1}$ grating).

\section{RESULTS AND DISCUSSION}

\section{Morphological evolution of GaN EHPs}

Given that the initial growth is determined by the shape of the SiN openings, the growth in circular openings results in GaN SHPs with six equivalent $\{1 \overline{1} 01\}$ planes, as a direct consequence of the hexagonal characteristics of the wurtzite crystal. ${ }^{11}$ The GaN SHP is formed by the volume enclosed by one (0001) surface and six equally sized $\{1 \overline{1} 01\}$ facets (Figure 1c). On the other hand, the growth in oval openings breaks the condition of equal size for all six $\{1 \overline{1} 01\}$ planes; Two of the six $\{1 \overline{1} 01\}$ planes are longer, determined by $E$, as depicted in Figure 1c. By prolonging the growth time, six $\{1 \overline{1} 01\}$ sidewalls converged to form an elongated (0001) surface directed along $E$ (forming a ridge). The length and width of the ridge were estimated to be approximately $1.0 \mu \mathrm{m}$ and $100 \mathrm{~nm}$, respectively, when $E$ was set to $1.0 \mu \mathrm{m}$. Owing to the sixfold symmetry of the wurtzite structure, rotating GaN EHPs grown from $\alpha=0^{\circ}$ with steps of $60^{\circ}\left(120^{\circ}\right)$ does not change the shape of GaN EHPs, but merely results in an in-plane rotation (cf. Figure 1d, upper part). However, a rotation with $\alpha=30^{\circ}, 90^{\circ}$ or $150^{\circ}$ leads to a shape different than that of $\alpha=0^{\circ}$. Assuming that the growth rate is similar for GaN EHPs and SHPs, and that the long axis of the GaN EHPs still follow $\alpha$, the growth of two planes perpendicular to $\alpha$ will be ceased during the growth. Continuous growth of the GaN EHPs will lead to small rhombic-shaped (0001) surfaces (Figure 1d, lower part). Consequently, one can say that the GaN EHPs with $\alpha=0^{\circ}, 60^{\circ}$ and $120^{\circ}$ are characterized by one type of morphology, while those with $\alpha=30^{\circ}$, $90^{\circ}$ and $150^{\circ}$ have another type of morphology. The orientations of the GaN EHPs as well the top ridges are found to be highly correlated with the polarizations directions of the emission from the InGaN QDs. Such polarization guiding will be further elucidated and verified in the following.

\section{Multiple InGaN QDs on a GaN EHP}

Figure 2a shows representative polarization-dependent microphotoluminescence $(\mu \mathrm{PL})$ spectra from a GaN EHP measured at $4 \mathrm{~K}$. A broad emission band peaking at $386 \mathrm{~nm}$ and several emission peaks in the range between 410 and $420 \mathrm{~nm}$ are observed. The monochromatic CL images in Figure $2 \mathrm{~b}$ clearly reveal that the broad emission band and the sharp peaks are associated with the InGaN layers on the $\{1 \overline{1} 01\}$ facets and the InGaN QDs on top of the GaN EHP, respectively. Such an observation is consistent with previous results, in which $c$-plane truncations on GaN SHPs serve as preferential QD formation sites via SK-like mechanism. ${ }^{11,26}$ The full-width half-maxima of the emission peaks in the present case are relatively broad $(>4 \mathrm{meV})$. Two additional experimental observations should be noted. First, spectral peaks with the fullwidth half-maxima as sharp as that of individual QDs on GaN SHPs (in the range of $0.35-1.2 \mathrm{meV}$ ) can be observed. ${ }^{11}$ Second, the peaks merge into a broad band when the number of QDs on a GaN EHP is increased by shortening the growth time of the pyramid to enlarge the top (0001) area. Therefore, the larger full-width half-maxima in the present case is suggested to be due to the inhomogeneous broadening caused by multiple QDs on top of the EHP. ${ }^{27}$ In addition, the emission peaks of InGaN QDs are not sensitive to the temperature. ${ }^{11,28}$ A temperature
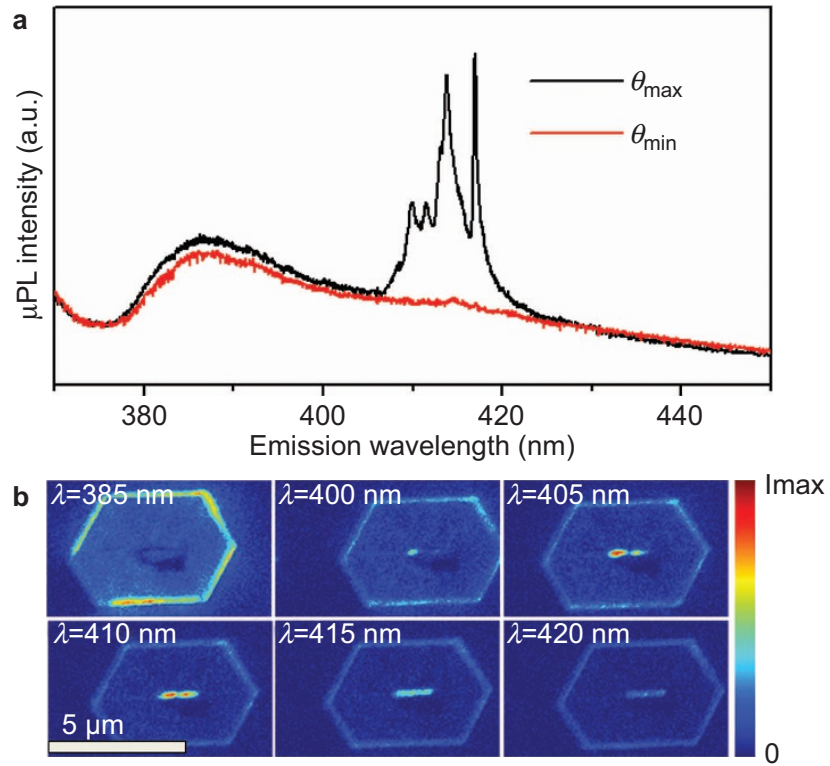

Figure 2 (a) $\mu \mathrm{PL}$ spectra of EHPs with the polarization analyzer set to $\theta_{\max }\left(\theta_{\min }\right)$ by which the maximum (minimum) intensity of sharp emission peaks are detected. (b) Spatially resolved monochromatic $\mathrm{CL}$ intensity maps of another GaN EHP from the same growth experiment. CL, cathodoluminescene; EHP, elongated hexagonal pyramid; $\mu \mathrm{PL}$, microphotoluminescence. 
robust optical behavior is advantageous for QD-based single photon emitters operating at high temperature.

Interestingly, all the QD-related emission peaks are strongly polarized along the same direction as evidenced by the fact that all peaks have their maximum and minimum intensities at the same $\theta$ (Figure 2a). Referring to our excitation/detection geometry, the linearly polarized emission can be ascribed to the in-plane anisotropy of the QD confinement potential. ${ }^{22,23}$ Thus, our experimental results imply that the in-plane anisotropies of all the InGaN QDs on the same EHP are simultaneously aligned. Moreover, the magnitude of in-plane anisotropy should be significant to achieve the observed high degree of polarization.

The polarization guiding effect of GaN EHPs

As shown in Figure 2a, all the emission peaks associated with the QDs on a GaN EHP are linearly polarized along the same direction (showing the same $\varphi$ ). Further correlation between the polarization-resolved measurements and the orientations of GaN EHPs (defined by $\alpha$ ) reveals that the polarization direction is parallel to the elongation $\left(\alpha \cong \varphi=0^{\circ}\right.$ in Figure 2a). As depicted in Figure 1, the implantation of $E$ in the mask design allows the creation of oval openings and the formation of GaN EHPs with ridges parallel to the $E$. The lengths of the ridges on GaN EHPs should be proportional to $E$, while the widths of the ridges should be independent on $E$, but related to the growth time. For simplicity, $E$ is used as an averaged parameter representing the degree of elongation in GaN EHPs. The effect of $E$ is statistically studied by measuring the polarization directions of QDs on GaN EHPs with various values of $E$ for the case of $\alpha=0^{\circ}$. As demonstrated in Figure 3, the polarization guiding effect becomes more effective for Elarger than $0.6 \mu \mathrm{m}$. A nearly perfect guiding effect (with a probability $>0.9$ ) is obtained for the maximal $E=1.0 \mu \mathrm{m}$ in the current mask design, implying that the elongated ridges help to guide the polarization. In addition, GaN EHPs with various values of $\alpha$ have been examined in order to investigate the possibility to control the polarization guiding in different directions. A polarization guiding $(\alpha \cong \varphi)$ is

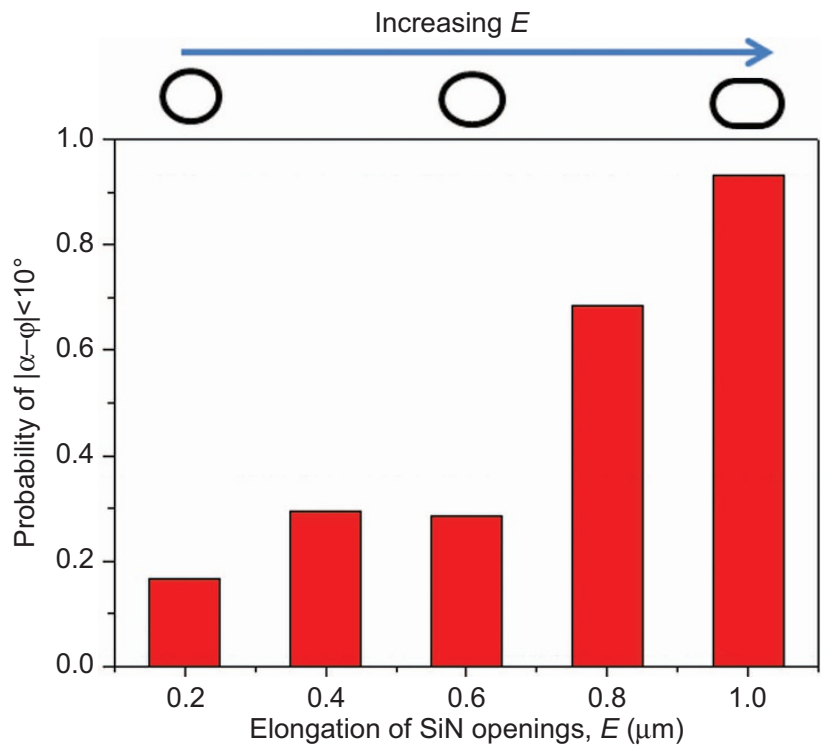

Figure 3 Statistical histogram of the observed polarization guiding effect as a function of $E$. The polarization guiding effect is defined in such a way that the measured polarization direction, $\varphi$, matches with the orientations of GaN EHPs, $\alpha$, satisfying the criterion $|\alpha-\varphi| \leq 10^{\circ}$. EHP, elongated hexagonal pyramid. $n=78$ for each different values of $E$. unambiguously revealed for GaN EHPs with $\alpha=0^{\circ}, 60^{\circ}$ and $120^{\circ}$ (as demonstrated in Figure 4). For the remaining group of GaN EHPs with $\alpha=30^{\circ}, 90^{\circ}$ and $150^{\circ}$, preferential polarization directions are seemly revealed, but $\alpha \cong \varphi$ is not strictly followed.

\section{The origin of polarization guiding}

To investigate the polarization guiding, the actual morphologies of those GaN EHPs shown in Figure 4 have been further examined. A general observation is that the GaN EHPs tend to comply with the design of the oval openings with $\alpha=0^{\circ}, 60^{\circ}$ and $120^{\circ}$, as evidenced by a large scale of uniform GaN EHPs shown in Figure 5a. The orientations of the GaN EHPs as well as the top ridges match well with the directions of the oval openings of $\alpha=0^{\circ}, 60^{\circ}$ and $120^{\circ}$ (compare the upper part of Figures $1 \mathrm{~d}$ and $5 \mathrm{~b}$ ). On the other hand, the growth of $\mathrm{GaN}$ EHPs with $\alpha=30^{\circ}, 90^{\circ}$ and $150^{\circ}$, does not lead to the formation of elongated ridges (Figure $5 \mathrm{c}$ ). It should be noted that the growth of EHPs with $\alpha=30^{\circ}\left(150^{\circ}\right)$ often resulted in distorted GaN EHPs with unidentified facets enclosed by the six $\{1 \overline{1} 01\}$ planes. Therefore, the polarization guiding effect can be ascribed to the orientations of $\mathrm{GaN}$ EHPs and the formation of elongated ridges.

Preferred polarization directions of III-N QDs grown on planar (0001)-oriented substrates have earlier been reported by using different fabrication approaches. ${ }^{22,23}$ In these reports, the preferential polarization directions were not understood. A unidirectional strain induced by the underlying layers to the overgrown QDs was given as a plausible explanation. However, such a behavior is a macroscopic character, rendering any further control of the polarization direction unrealistic based on current conventional growth techniques, as it would imply control of the strain under every QD over the entire substrate.

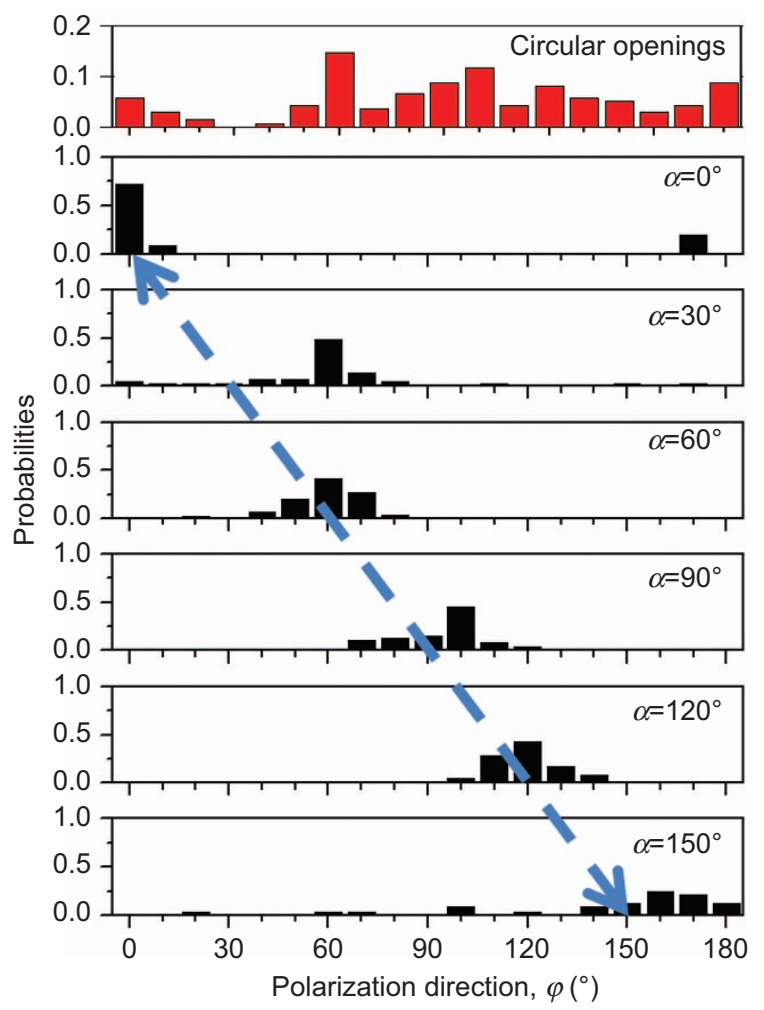

Figure 4 Distribution histograms of measured $\varphi$ from the GaN EHPs located in blocks with various values of $\alpha$. The results from reference GaN SHPs are also included for comparison ( $n=95)$. EHP, elongated hexagonal pyramid; SHP, symmetric hexagonal pyramid. $n=78$ for each different values of $\alpha$. 

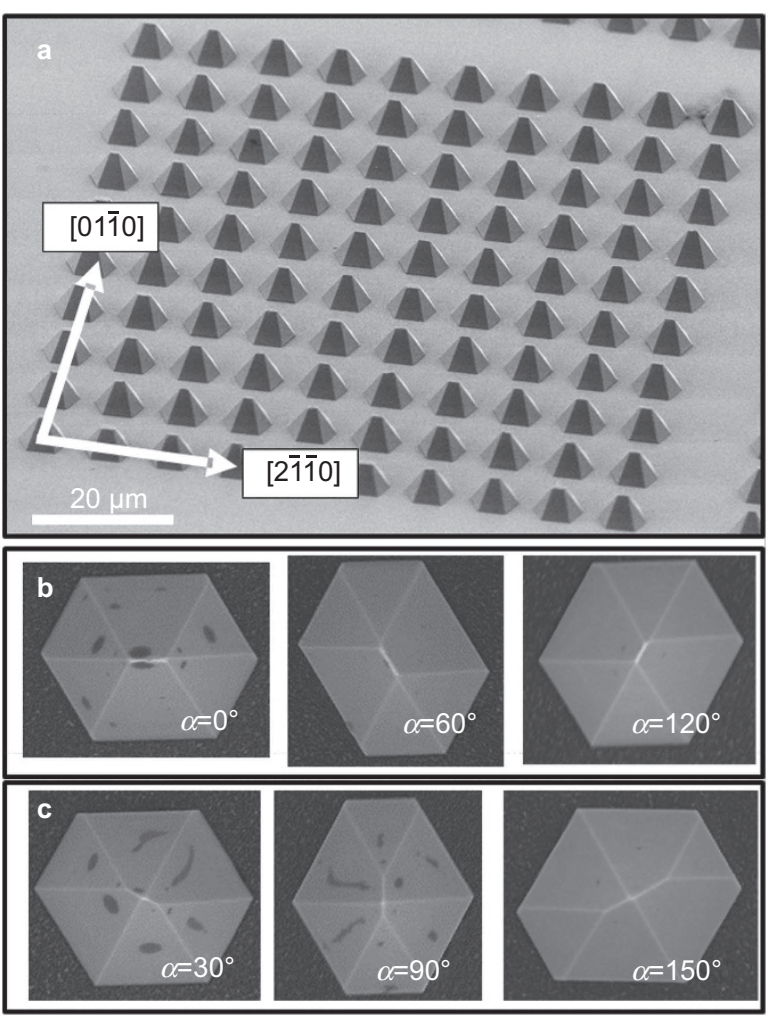

Figure 5 (a) SEM image showing uniform growth of GaN EHPs from oval openings with $\alpha=0^{\circ}$. (b, c) Magnified SEM images of individual GaN EHPs complied with oval openings with various values of $\alpha$. EHP, elongated hexagonal pyramid; SEM, scanning electron microscope.

The polarization control observed in this work can be explained in three ways: (i) the GaN EHPs transfer an anisotropic biaxial strain field to the QDs resulting in the formation of elongated QDs. The strength of the strain field in the EHPs should then be strongly correlated with $E$ and accordingly explaining why the guidance probability presented in Figure 3a increases continuously with $E$; (ii) given that the top parts of GaN EHPs are fully strain relaxed, as concluded for GaN SHPs, ${ }^{29}$ the asymmetry induced by a ridge will result in an anisotropic relaxation of the in-plane strain of the QDs on the ridge. The degree of relaxation is higher along the smallest dimension of the top area, i.e., along the direction perpendicular to the ridge elongation, leading to the ground state emission of the QD being polarized parallel to the ridge; and (iii) the ridge formation edges form a Schwoebel-Ehrlich barrier which prevents adatoms of diffusing out from the (0001) facet. ${ }^{30,31}$ Since the adatoms have larger probability to interact with an edge barrier parallel rather than orthogonal to the ridge elongation, the adatoms will preferentially diffuse parallel to the ridge elongation. Upon nucleation in such a directional diffusion of adatoms, elongated QDs may form. The polarization direction is known to be governed by the strain and shape anisotropy of the QD. As the strain and the shape of the QD are not independent factors and any accurate structural information of the QD currently is unavailable, further investigations are needed to gain additional information on the predominant factors determining the polarization.

\section{The high degree of polarization in III-N QDs}

Wherever the QDs are formed, on GaN SHPs or EHPs with various values of $E$, the degree of polarization for the QD emission is typically high, as shown in Figure 6a with measurements from a GaN EHP with $\alpha=0^{\circ}$. The polarization degree of the III-Ns is more sensitive to the inplane asymmetry compared to the III-As system due to the significant band mixing and the identical on-axis effective masses of the $\mathrm{A}$ and $\mathrm{B}$ bands in the III-N. ${ }^{22}$ A statistical investigation of the value of $P$ performed on $145 \mathrm{GaN}$ EHPs (Figure $6 \mathrm{~b}$ ) reveals that $93 \%$ of the investigated GaN EHPs possess $P>0.7$ with an average value of $P=0.84$. A similar investigation has been performed on GaN QDs grown on AlN/ Si (111) substrates by Bardoux et al. ${ }^{22}$ in which only $20 \%$ of the QD emissions had $P>0.7$ with an average value of $P=0.63$. The polarization of the emissions is related to the QD asymmetry determined by the anisotropy of the internal strain and electric fields, as well as by the structural shape of the QD itself. ${ }^{22}$ Numerical computations predict a high degree of polarization for small or moderate in-plane shape anisotropy of GaN and InGaN QDs. ${ }^{32,33}$ This is related to the intrinsic valence band structure of the III-Ns. To reach $P \cong 0.95$, an in-plane shape elongation of $1.25: 1$ is needed for an InGaN QD, ${ }^{23,33}$ while a corresponding aspect ratio of 2.5:1 is needed for GaN QDs. ${ }^{32}$ In particular, we have identified the split-off energy as the key material parameter determining the degree of polarization for a given asymmetry. ${ }^{21}$ Figure 7 shows the computed degree of polarization plotted
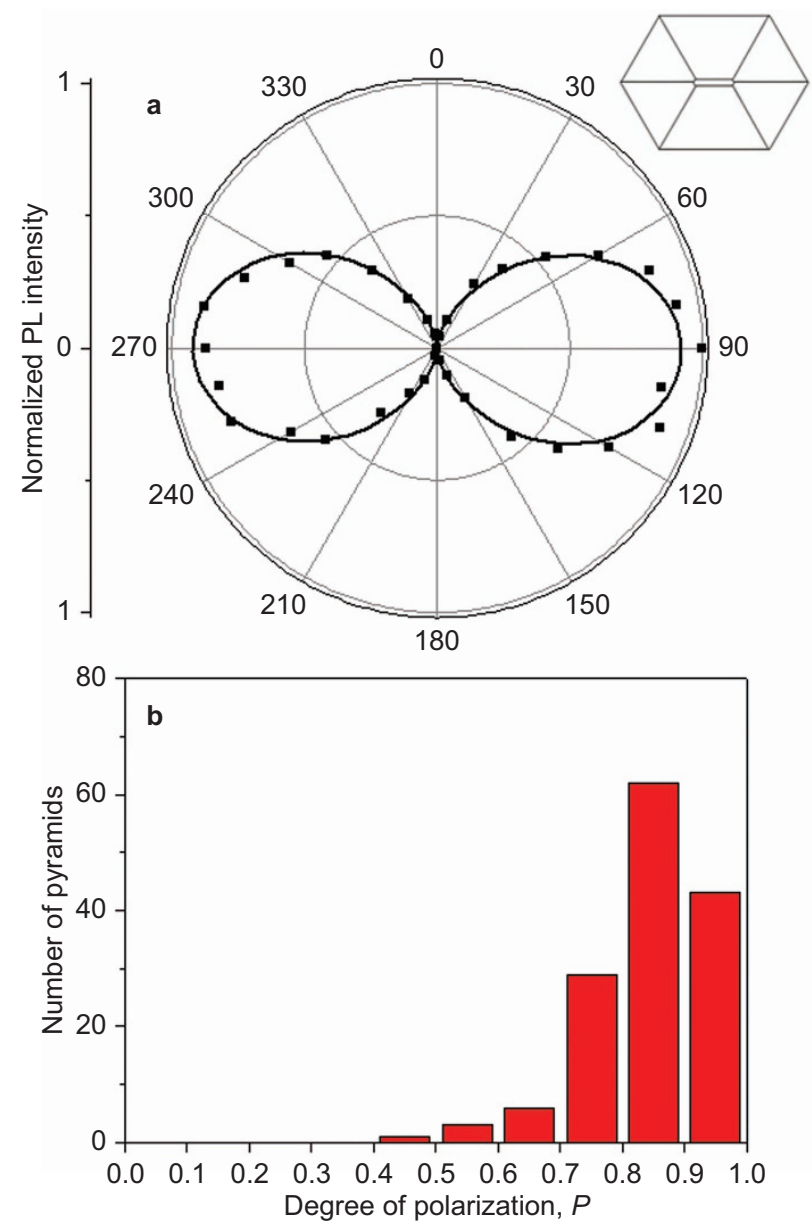

Figure 6 (a) Polar plots of the $\mu \mathrm{PL}$ intensity versus the angle of the polarization analyzer $(\theta)$ for three selected GaN EHPs with $\alpha=0^{\circ}$. (b) Statistical histogram showing the overall measured degree of polarization from GaN EHPs with $E=1 \mu \mathrm{m}$ and $\alpha=0^{\circ}, 60^{\circ}$ and $120^{\circ}$. Total number of pyramids measured is $n=234$ (78 for each different values of $\alpha$ ). EHP, elongated hexagonal pyramid; $\mu \mathrm{PL}$, microphotoluminescence; PL, photoluminescence. 


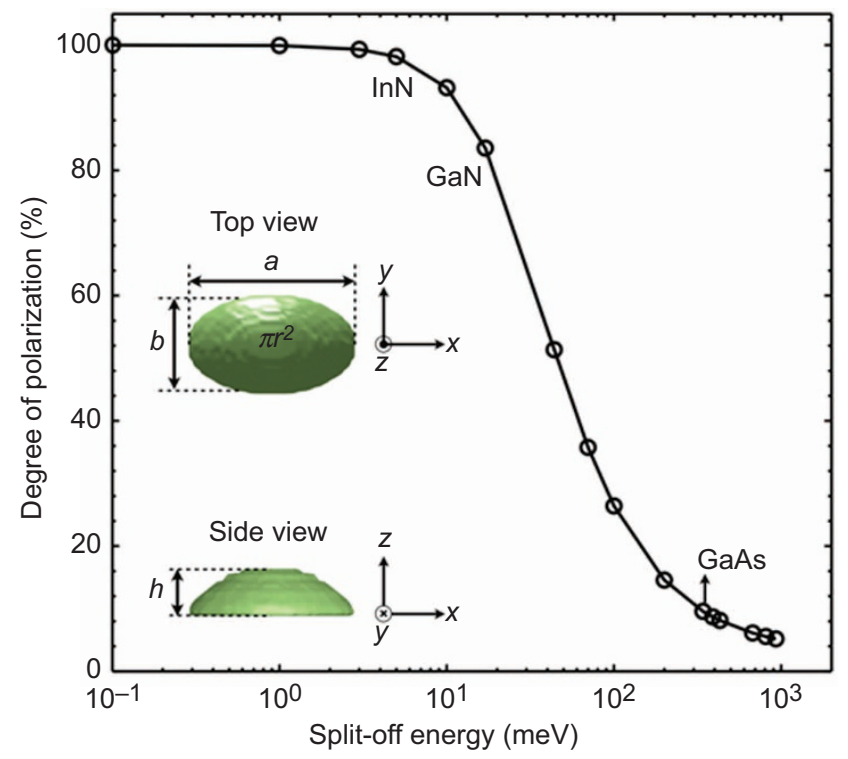

Figure 7 The computed degree of polarization plotted as a function of the split-off energy. The QD shape is assumed to be lens-shaped with an in-plane asymmetry of $b / a=0.8$. The single particle electron (hole) eigenstates are obtained from an effective mass Schrödinger equation (with a 6 band $\mathrm{k} \cdot \mathrm{p}$ Hamiltonian), discretized by finite differences. The Hamiltonians include strain and internal electric fields originating from spontaneous and piezoelectric polarizations. The polarized optical transitions are computed by the dipole matrix elements. QD, quantum dot.

against a variation of the split-off energy. Given a fixed asymmetry of the QDs, it is concluded that the material with the smallest split-off energy exhibits the highest degree of polarization. The extreme sensitivity on the asymmetry for InGaN QDs can be ascribed to a significant band mixing in InN and implies its inherent advantage for the generation of photons possessing a specific polarization.

\section{CONCLUSIONS}

In this paper, we have demonstrated an effective method to achieve site-controlled QDs emitting linearly polarized emission with controlled polarization directions by growing InGaN QDs on top of elongated GaN pyramids in a metal organic chemical vapor deposition system. The polarization directions of the QD emission can be guided by the orientations of the underlying elongated GaN pyramids. Such an effect can be realized as the elongated GaN pyramids provide additional in-plane confinement for the InGaN QDs implanting unidirectional in-plane anisotropy into the QDs, which subsequently emit photons linearly-polarized along the elongated direction of the GaN EHPs. Several advantages of our present work can be expected for applications. First, the deep confinement potential which is achievable for III-N QDs will allow high temperature operation. ${ }^{20}$ Second, the emission wavelength regime could be tunable from ultraviolet to near infrared by adjusting the In composition in the InGaN. Third, a high polarization degree is more easily achieved in InGaN QDs compared to other semiconductor materials, even if the crystalline orientation is [0001]. Otherwise, semipolar or non-polar III-N layers must be used to achieve a high polarization degree..$^{34}$ Fourth, our approach is compatible with current large-area wafers and processing techniques. ${ }^{35}$ If the number of QDs on a GaN EHPs is reduced, site-controlled QDs with controlled separation will facilitate the fabrication of ultracompact single-photon emitters with controllable photon polarization vectors on a single chip.

\section{AUTHOR CONTRIBUTIONS}

$\mathrm{AL}$ and $\mathrm{CWH}$ designed and performed the experiments, meanwhile analyzed the results and wrote the manuscript. KFK guided the optical investigations, conducted the numerical calculations and edited the manuscript. SA analyzed the results of the numerical calculations. DN performed the cathodoluminescence measurements. UF guided the material growth in metal organic chemical vapor deposition. EJ and $\mathrm{POH}$ guided the project and finalized the manuscript.

\section{ACKNOWLEDGMENTS}

This work was mainly supported by the Nano-N consortium funded by the Swedish Foundation for Strategic Research. We also acknowledge support from the Swedish Government Strategic Research Area in Materials Science on Functional Materials at Linköping University (Faculty Grant SFO-Mat-LiU \# 2009-00971) and grants from the Swedish Research Council (VR) and the Knut and Alice Wallenberg Foundation. The authors want to thank Jan-Ake Larsson (ISY, Linköping University), Iryna Yakymenko (IFM, Linköping University) and Marc-Andre Dupertuis (EPFL, Laboratory of Physics of Nanostructures, Switzerland) for fruitful discussions.

1 Arakawa Y. Progress in growth and physics of nitride-based quantum dots. Phys Status Solidi (a) 2001; 188: 37-45.

2 Shields AJ. Semiconductor quantum light sources. Nat Photon 2007; 1: 215-223.

3 Zeng $\mathrm{N}$, Jiang $\mathrm{X}, \mathrm{Gao} \mathrm{Q}, \mathrm{He} \mathrm{Y}, \mathrm{Ma} \mathrm{H}$. Linear polarization difference imaging and its potential applications. Appl Opt 2009; 48: 6734-6739.

4 Knill A, Laflamme R, Milburn GJ. A scheme for efficient quantum computation with linear optics. Nature 2001; 409: 46-52.

5 Young RJ, Ellis DJ, Stevenson RM, Bennett AJ, Atkinson P et al. Quantum-dot sources for single photons and entangled photon pairs. Proc IEEE 2007; 95: 1805-1814.

6 Ishikawa T, Nishimura T, Kohmoto S, Asakawa K. Site-controlled InAs quantum-dot structures on GaAs surfaces patterned by in-situ electron beam lithography. Appl Phys Lett 2000; 76: 167-169.

7 Hartmann A, Ducommun Y, Loubies L, Leifer K, Kapon E. Structure and photoluminescence of single $\mathrm{AlGaAs} / \mathrm{GaAs}$ quantum dots grown in inverted tetrahedral pyramids. Appl Phys Lett 1998; 73: 2322-2324.

8 Chithrani D, Williams RL, Lefebvre J, Poole PJ, Aers GC. Optical spectroscopy of single site-selected InAs/InP self-assembled quantum dots. Appl Phys Lett 2004; 84: 978980.

9 Edwards PR, Martin RW, Watson IM, Liu C, Taylor RA et al. Quantum dot emission from site-controlled InGaN/GaN micropyramid arrays. Appl Phys Lett 2004; 85 : 4281-4283.

10 Chithrani D, Williams RL, Lefebvre J, Poole PJ, Aers GC. Optical spectroscopy of single, site-selected, InAs/InP self-assembled quantum dots. App/ Phys Lett 2004; 84: 978-980.

11 Hsu CW, Lundskog A, Karlsson KF, Forsberg U, Janzén E et al. Single excitons in InGaN quantum dots on GaN pyramid arrays. Nano Lett 2011; 11: 2415-2418.

12 Minot ED, Kelkensberg F, van Kouwen M, van Dam JA, Kouwenhoven LP et al. Single quantum dot nanowire LEDs. Nano Lett 2007; 7: 367-371.

13 Choi K, Arita M, Kako S, Arakawa Y. Site-controlled growth of single GaN quantum dots in nanowires by MOCVD. J Cryst Growth 2013; 370: 328-331.

14 Deshpande S, Heo J, Das A, Bhattacharya P. Electrically driven polarized singlephoton emission from an InGaN quantum dot in a GaN nanowire. Nat Commun 2013; 4: 1675 .

15 Kita T, Wada O, Ebe H, Nakata Y, Sugawara M. Polarization-independent photoluminescence from columnar InAs/GaAs self-assembled quantum dots. Jpn J Appl Phys 2002; 41: L1143-L1145.

16 Yasuoka N, Ebe H, Kawaguchi K, Ekawa M, Morito K et al. Polarization-insensitive quantum dot semiconductor optical amplifiers using strain-controlled columnar quantum dots. J Lightwave Technol 2012; 30: 68-75.

17 Strauf S, Stoltz NG, Rakher MT, Coldren LA, Petroff PM et al. High-frequency single photon source with polarization control. Nat Photon 2007; 1: 704-708.

18 Unitt DC, Bennett AJ, Atkinson P, Ritchie DA, Shields AJ. Polarization control of quantum dot single-photon sources via a dipole-dependent Purcell effect. Phys Rev B 2005; 72: 033318.

19 Rakher MT, Stoltz NG, Coldren LA, Petroff PM, Bouwmeester D. Polarizationswitchable single photon source using the Stark effect. App/ Phys Lett 2008; 93 : 091118.

20 Kako S, Santori C, Hoshino K, Gotzinger S, Yamamoto Y et al. GaN single photon source operating at 200K. Nat Mater 2006; 5: 887-892.

21 Amloy S, Karlsson KF, Holtz PO. III-nitride based quantum dots for photon emission with controlled polarization switching. arXiv: 1311.5731: http://arxiv.org/abs/ 1311.5731. 
22 Bardoux R, Guillet T, Gil B, Lefebvre P, Bretagnon T et al. Polarized emission from GaN/AIN quantum dots: single-dot spectroscopy and symmetry-based theory. Phys $\operatorname{Rev} B$ 2008; 77: 235315.

23 Winkelnkemper M, Seguin R, Rodt S, Schliwa A, Reißmann L et al. Polarization emission lines from A- and B-type exciton complexes in single InGaN/GaN QDs. J Appl Phys 2007; 101: 113708.

24 Forsberg U, Lundskog A, Kakanakova-Georgieva A, Ciechonski R, Janzén E. Improved hot-wall MOCVD growth of highly uniform AIGaN/GaN/HEMT structures. J Cryst Growth 2009; 311: 3007-3010.

25 Lundskog A, Forsberg U, Holtz PO, Janzén E. Morphology control of hot-wall MOCVD selective-area-grown hexagonal GaN pyramids. Cryst Growth Des 2012; 12: 5491-5496.

26 Lundskog A, Palisaitis J, Hsu CW, Eriksson M, Karlsson KF et al. InGaN quantum do formation mechanism on hexagonal GaN/InGaN/GaN pyramids. Nanotechnology 2012; 23: 305708.

27 Empedocles SA, Bawendi MG. Quantum-confined stark effect in single CdSe nanocrystallite quantum dots. Science 1997; 278: 2114-2117.

28 Hsu CW, Moskalenko ES, Eriksson MO, Lundskog A, Karlsson KF et al. The charged exciton in an InGaN QD on a GaN pyramid. App/ Phys Lett 2013; 103: 013109.

29 Liu QK, Hoffmann A, Siegle H, Kaschner A, Thomsen C et al. Stress analysis of selective epitaxial growth of GaN. App/ Phys Lett 1999; 74: 3122-3124.
30 Pierre-Louis O, D'Orsogna MR, Einstein TL. Edge diffusion during growth: the kink Schwoebel-Erhlich effect and resulting instabilities. Phys Rev Lett 1999; 82: 36613664.

31 Liu SJ, Wang EG, Woo CH, Huang H. Three-dimensional Schwoebel-Ehrlich barrier. J Comp Aid Mater Des 2001; 7: 195-201.

32 Amloy S, Karlsson KF, Andersson TG, Holtz PO. On the polarized emission from exciton complexes in GaN quantum dots. App/ Phys Lett 2012; 100: 021901.

33 Winkelnkemper M, Schliwa A, Bimberg D. Interrelation of structural and electronic properties in $\operatorname{In}_{x} \mathrm{Ga}_{1-x} \mathrm{~N}$ quantum dots using an eight-band $\mathrm{k} \cdot \mathrm{p}$ model. Phys Rev $B$ 2006; 74: 155322 .

34 Matioli E, Brinkley S, Kelchner KM, Hu YL, Nakamura S et al. High-brightness polarized light-emitting diodes. Light Sci Appl 2012; 1: e22; doi:10.1038/ Isa.2012.22.

35 Choi JH, Zoulkarneev A, Kim SI, Baik CW, Yang MH et al. Nearly single-crystalline GaN light-emitting diodes on amorphous glass substrates. Nat Photon 2011; 5: 763-769.

(c) (i) $\odot$ This work is licensed under a Creative Commons Attribution-

(c) this license, visit http://creativecommons.org/licenses/by-nc-nd/3.0 